\title{
COMPOSICIÓN FLORISTICA DE LOS BOSQUES SECUNDARIOS DE JENARO HERRERA, LORETO
}

\author{
Juan Baluarte Vásquez *
}

\section{RESUMEN}

Los bosques secundarios de origen antrópico en Jenaro Herrera cuentan con más de 30 años de establecidos; sin embargo, han sido escasamente estudiados en comparación con otras zonas tropicales del país y del mundo. Los bosques secundarios están considerados como márgenes de bosques que actúan como barreras para contrarrestar el avance de la agricultura de subsistencia. No obstante su importancia, los bosques secundarios en Jenaro Herrera han sido raramente estudiados conociéndose muy poco sobre su composición florística. Con este estudio se ha cubierto en parte este vació de información. La composición florística de los bosques secundarios en Jenaro Herrera está caracterizada por contar con un reducido grupo de familias con mayor abundancia de especies: Fabaceae 10\%, Melastomaceae 9\%, Rubiaceae 5\%, Annonaceae 5\%, Arecaceae 4,5\%, Clusiaceae 4\%, Moraceae 4\% y Euphorbiaceae 3\% Cecropiaceae $2.5 \%$ y Piperacea 2\%. Las familias Melastomácea, Cecropiácea, Rubiácea y Euphorbiáeea congregan más del $50 \%$ de plantas de los 880 individuos evaluados. Los géneros con mayor abundancia de especies son Miconia (12 especies), Ínga (7 especies), Vismia (4 especies), Palicourea (4 especies) y Guatteria (4 especies). Existen 15 especies comunes que aparecen en más de cuatro levantamien- 2 tos, siendo las más frecuentes: Paviana aff. campestris (en siete levantamientos); Alchornea triplinervia, Leandra secunda y Miconia minutiflora (en seis levantamientos).

Palabras clave: Bosque secundario (terraza alta), composición florística, Jenaro Herrera, Amazonía Peruana. 


\begin{abstract}
The secondary forests of antropic origin in Jenaro Herrera are over 30 years oid; however, they have hardly been studied if we compare them with other tropical areas in the country and the world. The secondary forests are considered as borderlines of forest and serve as a barrier to prevent agriculture from advancing. Despite their importance, the secondary forests in Jenaro Herrera have rarely been studied. Little is known of its floweristjc composition. This study has helped cover this part of the information gap. The floweristic composition in the secondary forests in Jenaro Herrera comprises a small group of families, standing aut: Fabaceae 10\%; Melastomaceae 9\%; Rubiaceae 5\%, Annonaceae 5\%; Arecaceae 4,5\%. Clusiaceae 4\% Moraceae 4\% Euphorbiaceae 3\% Cacropiaceae 2,5\% y Piperaceae 2\% species and Miconia (12 species); Inga (7 species); Visrnia (4 species); Palicourea (4 species) y Guatteria (4 species).
\end{abstract}

There are 15 common species that are found in four arcas, being the most comrnon: Pariana aff. cainpestris (in seven arcas); Alchornea triplinervia, Leandra secunda and Miconia ninutiflora (in six areas).

Key words: Secondary forest (high terrace or tableau), floweristic composition, Jenaro Herrera, Peruvian Amazon Region.

\title{
1. INTRODUCCION
}

En 1965 se inició el proyecto Asentamiento Rural Integral Jenaro Herrera financiado por la Cooperación Técnica Suiza (COTESU), para promover el desarrollo rural de la zona basado en la ganadería y la actividad forestal. En virtud de ello, se convirtieron extensas áreas de bosques en pastos para la alimentación de ganado vacuno.

El retiro de la COTESU aceleró el fracaso de este modelo de desarrollo. Las parcelas dedicadas a pastos se abandonaron, dando paso a la vegetación secundaria.

A pesar de haber transcurrido más de 30 años, los estudios sobre bosques secundarios en Jenaro Herrera son muy escasos en comparación a otras zonas tropicales del país y del mundo. 
Con este estudio se pretende cubrir en parte ese vacío de información, aportando conocimientos sobre su composición florística para contribuir con el manejo de estos.

bosques. Sin embargo, debido a la ausencia de vegetaciones secundarias antiguas, se ha limitado el estudio a poblaciones de uno hasta 17 años de desarrollo.

Para tal efecto, se seleccionó vegetaciones secundarias de 1, 3, 5, 8, 10, 12, 14 y 17 años; instalando en cada una de ellas parcelas de muestreo de $20 \mathrm{~m}$ x $50 \mathrm{~m}$, conteniendo en su interior sub-parcelas de $2,5 \mathrm{~m}$ x 2,5 $\mathrm{m}$ y $5 \mathrm{~m}$ x $5 \mathrm{~m}$, encuadradas en tres sub-parcelas de $10 \mathrm{~m}$ x $10 \mathrm{~m}$, ubicadas en los extremos y parte central de la parcela mayor; donde se evaluaron plantas de diferentes tamaños y diámetros. En cada una de estas unidades de muestreo se efectuó el levantamiento de la vegetación, acompañado de la colecta del material botánico.

\section{MATERIALES Y METODOS}

\subsection{Descripción de la zona de estudio}

El trabajo de campo se efectuó en los bosques secundarios de la terraza alta de Jenaro Herrera ( $73^{\circ} 40^{\prime} \mathrm{y}^{\circ} 45^{\prime}$ ' S), a $200 \mathrm{~km}$ de Iquitos en dirección suroeste y a $125 \mathrm{msnm}$.

Los registros climáticos muestran promedios de temperatura, precipitación total anual y humedad relativa de $25,9^{\circ} \mathrm{C}, 2889 \mathrm{~mm}$ y $87 \%$, respectivamente.

Según ONERN (1976), el área de influencia de la zona de estudio está clasificada como Bosque Húmedo Tropical (bh-T). La zona se caracteriza por presentar terrenos de relieve suavemente ondulado, con pendientes de $5 \%$ a $15 \%$ (Freitas, 1985). Estas pendientes forman pequeñas quebradas que se desbordan cuando llueve.

Los suelos predominantes son muy ácidos y presentan contenidos bajos de nutrientes con alta saturación de Al y bajo contenido de Ca (Weel, 1985).

\subsection{Materiales y métodos}

\subsubsection{Selección y ubicación de las purmas}

Para la selección de las purmas de diferentes edades de desarrollo se efectuó consultas a los productores de la localidad. De esta manera se 
escogieron ocho purmas de 1, 3, 5, 8, 10, 12, 14, y 17 años; todas ubicadas entre 2,5 y $6,5 \mathrm{~km}$ del eje de la carretera Jenaro Herrera Colonia Angamos.

\subsubsection{Forma y tamaño de las parcelas}

Para la evaluación de la vegetación, se utilizó el método de parcelas incluidas (Carvalho et al., 1986; UNALM, 1987 y Flores, 1990) ubicando dentro de la parcela mayor, correspondiente a un rectángulo de $20 \mathrm{~m} \mathrm{x}$ $50 \mathrm{~m}(0,1 \mathrm{ha})$, sub-parcelas de $10 \mathrm{mx} 10 \mathrm{~m}, 5 \mathrm{mx} 5 \mathrm{~m}$ y $2,5 \mathrm{~m}$ x $2,5 \mathrm{~m}$ para la evaluación de plantas de diversas alturas y diámetros (Fig.1).

\subsubsection{Evaluación de la vegetación}

El levantamiento de la vegetación se efectuó tomando como punto de partida una de las sub-parcelas del extremo, evaluando primero la sub-parcela de 2,5 x 2,5 m, continuando con la de $5 \mathrm{~m}$ x $5 \mathrm{~m}$, para culminar luego con la de $10 \mathrm{~m}$ x $10 \mathrm{~m}$. Así, una vez concluida esta sub-parcela, se evaluó la subparcela central, repitiendo este mismo procedimiento con la sub -parcela del otro extremo.

Cada una de las plantas evaluadas fueron colectadas para su posterior identificación. Cuando se presentaba más de una planta similar se procedía a la colección del material botánico más representativo, siempre y cuando se tenía la seguridad de que se trataba del mismo taxón.

Cada planta evaluada fue registrada en el cuaderno de campo con los siguientes datos: número de colecta correlativa, edad de la purma y forma biológica.

\subsubsection{Tratamiento taxonómico}

El primer nivel de identificación de las plantas se realizó en el campo. Con el objeto de dar mayor seguridad a la identificación, se colectaron muestras botánicas.

Las muestras botánicas debidamente herborizadas fueron trasladadas al herbario de la Universidad Nacional Mayor de San Marcos para su identificación, lugar en donde se encuentran clasificadas.

El primer paso de la identificación consistió en agrupar las muestras a nivel de morfoespecie, seguidamente se recurrió al uso de claves basadas en 
órganos reproductivos de Gentry (1991) y flórulas (Spichiger et al., 1989, 1990).

Gran parte de las muestras colectadas no contaron con órganos reproductivos al momento de la evaluación, razón por la cual se tuvo que recurrir para su identificación a claves basadas en órganos vegetativos de Spichiger (1982) y Kahn (1990). La comparación con muestras botánicas del herbario fue también un recurso muy útil en la identificación. Para la identificación de los helechos se utilizó la clave de Stolze (1981), Tryon \& Stolze (1989) y Tryon et al. (1991).

Para la clasificación taxonómica y la comprobación de la nomenclatura correcta de las especies, además de las floras y flórulas antes mencionadas, se revisó el catálogo de plantas Angiospermas y Gimnospermas del Perú de Brako y Zarucchi (1993).

\section{RESULTADOS Y DISCUSION}

La composición florística de la vegetación secundaria de Jenaro Herrera ha sido poco estudiada, razón por la cual los resultados obtenidos en este estudio serán también comparados con los de otras zonas tropicales del país y del mundo.

Las parcelas evaluadas están constituidas por 200 especies, 132 géneros y 62 familas. Estos taxones están distribuidos en forma aleatoria en las diferentes vegetaciones secundarias evaluadas.

La Figura 2 ilustra la forma como los taxones se distribuyen a través de las diferentes vegetaciones evaluadas, observándose de manera general una tendencia de incremento del número de familias, géneros y especies en las vegetaciones de mayor edad, aspecto que también fue observado por Weel (1985) en Jenaro Herrera y Budowski (1985) y Ríos (1990) en otras zonas tropicales del país y el mundo.

Un reducido número de familias (10 taxones) contienen cerca del 50\% de las especies de las vegetaciones evaluadas (Figura 3). Esto nos permite aseverar que existe una alta homogeneidad florística en la vegetación estudiada; este aspecto también fue observado por la Universidad Nacional Agraria La Molina.UNALM(1987) en los bosques de San Alejandro, Pucallpa. 
Cuatro familias (Melastomácea, Cecropiácea, Rubiácea y Euphorbiácea) congregan más del $50 \%$ de plantas de los 880 individuos evaluados. Esta particularidad se resalta en la Figura 4. La inclusión en este grupo de Cecropiáceas y Euphorbiáceas, que cuentan con pocas especies, es una demostración que no siempre el mayor número de especies implica una mayor abundancia de individuos. Asimismo, en estas cuatro familias la forma biológica predominante está representada por arbustos y árboles.

Los géneros con mayor abundancia de especies son Miconia (12 especies), higa (7 especies), Vismia (4 especies), Palicourea (4 especies) y Guatteria (4 especies); ver Cuadro 2 del Anexo.

La relación de todas las especies evaluadas por levantamiento se incluye en el Cuadro 2; allí se observa algunas especies comunes en las vegetaciones de diferentes edades de desarrollo. Así, cinco de las 33 especies que crecen en la vegetación de tres años también se presentan en la vegetación secundaria de un año. En la vegetación secundaria de cinco años se observa cinco especies que son frecuentes en la vegetación secundaria de un año y ocho con la de tres años. De éstas, dos son comunes en las parcelas de uno, tres y cinco años.

Igualmente, en la vegetación secundaria de ocho años se observa que seis especies son similares con la vegetación secundaria de un año, ocho con la de tres años e igual número con la de cinco años. La vegetación secundaria de diez años presenta también algunas especies afines; así, tres son frecuentes con la vegetación de un año, seis con la de tres años, 11 con la de cinco años y seis con la de ocho años. La vegetación secundaria de 12 años presenta también cuatro especies comunes con la vegetación de un año, ocho con la vegetación de tres años, siete con la vegetación de cinco años, cinco con la de ocho años y 13 con la de 10 años.

Asimismo, 6 especies de la vegetación secundaria de 14 años se repiten en la vegetación de un año; de igual modo, 10 especies de esta misma vegetación son comunes con la de 3 años, 8 con la de 5 años, 5 con la de 8 años, 10 con la de 10 años y 9 con la de 12 años. De igual modo, 5 especies de la vegetación secundaria de 17 años son concurrentes con la vegetación de un año, 4 con la de 3 años, 8 con la de 5 años, 6 con la de 8 años, 15 con la de 10 años, 10 con la de 12 años y 9 con la de 14 años.

En todas las vegetaciones evaluadas, se aprecia la existencia de un conjunto de 15 especies presentes en más de cuatro levantamientos; de éstas siete son árboles, seis son arbustos, una es liana y otra es herbácea (Cuadro 1). 


\section{Cuadro 1. Relación de especies más abundantes.}

\begin{tabular}{|c|c|c|c|c|c|c|c|c|c|c|}
\hline \multirow[t]{2}{*}{ ESPECIE } & \multirow[t]{2}{*}{ FAMILIA } & \multirow{2}{*}{$\begin{array}{l}\text { FORMA } \\
\text { BIOLÓGICA / } \\
\text { ESTRATO } \\
\text { OCUPACIONAL }\end{array}$} & \multicolumn{8}{|c|}{ EDAD DE LA PURMA (Años) } \\
\hline & & & 1 & 3 & 5 & 8 & 10 & 12 & 14 & 17 \\
\hline \multirow{14}{*}{$\begin{array}{l}\text { AegiphiIa aff. spicata } \\
\text { Alchornea triplinervia } \\
\text { Bactris sp. } \\
\text { Casearia arbórea } \\
\text { Casearia javitensis } \\
\text { Cecropia ficifolia } \\
\text { Ladenbergia sp. } \\
\text { Leandra secunde } \\
\text { Miconia minutiflora } \\
\text { Miconia niyriantha } \\
\text { Ocotea oblonga } \\
\text { Pariana aff. campestris } \\
\text { Psychotri idotricha } \\
\text { Tococa guien enszs } \\
\text { Vismia gracilis }\end{array}$} & \multirow{14}{*}{$\begin{array}{l}\text { Verbenaceae } \\
\text { Euphorbiae } \\
\text { Arecaceae } \\
\text { Flacourtiac. } \\
\text { Flacourtiac. } \\
\text { Cecropiaceae } \\
\text { Rubiaceae } \\
\text { Melastomae } \\
\text { Melastomae } \\
\text { Melastomae } \\
\text { Lauraceae } \\
\text { Poaceae } \\
\text { Rubiaceae } \\
\text { Melastomae } \\
\text { Clusiaceae }\end{array}$} & \multirow{14}{*}{$\begin{array}{l}\text { liana/medio } \\
\text { árbol/sup. } \\
\text { arbust/med. } \\
\text { arbust/med. } \\
\text { arbust/med. } \\
\text { árbol/sup. } \\
\text { árbol/med. } \\
\text { arbust/med. } \\
\text { árbollsup. } \\
\text { árbol/medio } \\
\text { árbol/medio } \\
\text { herbac/inf. } \\
\text { arbust/med. } \\
\text { arhsut/med. } \\
\text { arhsut/medio }\end{array}$} & $\mathrm{x}$ & $\mathrm{x}$ & $\mathrm{x}$ & & $\mathrm{x}$ & & & \\
\hline & & & & $\mathrm{x}$ & $\mathrm{x}$ & & $\mathrm{x}$ & $\mathrm{x}$ & $\mathrm{x}$ & $\mathrm{x}$ \\
\hline & & & $\mathrm{x}$ & $\mathrm{x}$ & & $\mathrm{x}$ & & & $\mathrm{x}$ & $\mathrm{x}$ \\
\hline & & & & & & & $\mathrm{x}$ & & $\mathrm{x}$ & $\mathrm{x}$ \\
\hline & & & $\mathrm{x}$ & & $\mathrm{x}$ & $\mathrm{x}$ & & & $\mathrm{x}$ & $\mathrm{x}$ \\
\hline & & & & $\mathrm{x}$ & & & $\mathrm{x}$ & $\mathrm{x}$ & $\mathrm{x}$ & $\mathrm{x}$ \\
\hline & & & $\mathrm{x}$ & $\mathrm{x}$ & & $\mathrm{x}$ & $\mathrm{x}$ & $\mathrm{x}$ & $\mathrm{x}$ & \\
\hline & & & $\mathrm{X}$ & & $\mathrm{x}$ & $\mathrm{x}$ & $\mathrm{x}$ & & $\mathrm{x}$ & $\mathrm{x}$ \\
\hline & & & $\mathrm{x}$ & & & $\mathrm{x}$ & $\mathrm{x}$ & $\mathrm{x}$ & & \\
\hline & & & & $\mathrm{x}$ & $\mathrm{x}$ & $\mathrm{x}$ & $\mathrm{x}$ & & $\mathrm{x}$ & \\
\hline & & & $\mathrm{x}$ & $\mathrm{x}$ & $\mathrm{x}$ & & $\mathrm{x}$ & $\mathrm{x}$ & $\mathrm{X}$ & $\mathrm{X}$ \\
\hline & & & & $\mathrm{x}$ & & $\mathrm{x}$ & & $\mathrm{x}$ & & $\mathrm{X}$ \\
\hline & & & & & $\mathrm{X}$ & $\mathrm{x}$ & $\mathrm{x}$ & & $\mathrm{x}$ & \\
\hline & & & $\mathrm{X}$ & $\mathrm{x}$ & $\mathrm{X}$ & & & & $\mathrm{X}$ & \\
\hline
\end{tabular}

Donde: X significa ocurrencia de la especie.

La especie más frecuente, que está presente en el $88 \%$ de los levantamientos, es Pariana aif. campestris. Esta solamente está ausente en la vegetación de ocho años. Asimismo, las especies Alchornea triplinervia, Leandra secunda y Miconia minutiflora, ocurren en seis levantamientos, siendo también consideradas como las especies más comunes de las vegetaciones secundarias evaluadas.

En términos generales, la composición florística de la vegetación secundaria evaluada es casi similar a los resultados obtenidos por Weel (1985) en Jenaro Herrera - Iquitos y Ríos (1990) en San Alejandro - Pucallpa, por lo menos a nivel genérico. Al respecto, UNESCO, PNUMA y FAO (1980), señalan que las semejanzas entre los bosques secundarios jóvenes de las diferentes regiones tropicales son llamativas y agregan también que Cecropia, de las formaciones secundarias jóvenes de América tropical húmeda, desempeña el mismo papel que Musanga cecropiodes, de las de Africa; esta observación fue también resaltada por Kahn (1983). 


\section{CONCLUSIONES}

- La composición florística del área estudiada está conformada por 200 especies pertenecientes a 132 géneros y 62 familias.

- La composición florística está caracterizada por contar con un reducido grupo de familias con mayor abundancia de especies: Fabaceae 10\%, Melastomaceae $9 \%$, Rubiaceae 5\%, Annonaceae 5\%, Arecaceae 4,5\%, Clusiaceae 4\%, Moraceae ${ }_{400}$ y Euphorbiaceae 3\%, Cecropiaceae 2,500 y Piperaceae 2\%.

- Las familias Melastomácea, Cecropiácea, Rubiácea y Euphorbiácea congregan más del $50 \%$ de plantas de los 880 individuos evaluados.

- Los géneros con mayor abundancia de especies son Miconia (12 especies), Inga (7 especies), 14smia (4 especies), Palicourea (4 especies) y Guatteria (4 especies).

- Existen 15 especies comunes que aparecen en más de cuatro levantamientos, siendo las más frecuentes: Pariana aff. carnpestris (en siete levantamientos); A icho rn ea trzplinervia, Leandra secunda yMiconia minutzflora (en seis levantamientos).

- Las especies más representativas de los diferentes estratos, en orden de abundancia, son: (a) estrato inferior: Pariana aff. carnpestris; (b) estrato medio: Leandra secunda, Ladenbergia sp., Aegiphilla aif. spicata, Tococa guianensis, Casearia arborea, Visrnia graciiis, Psichotria iodotricha, Caseariajavitensis y Miconia myriantha; (e) estrato superior: Cecropiafic folia, Miconia niinutzflora y Alchornea tripíinervia.

\section{BIBLIOGRAFIA}

BRAKO, L., ZARUCCHJ, J. 1993. Catalogue of the Fíowering plants and Gimnosperrns of Perú. St. Louis, Missouri (Estados Unidos): Missouri Botanical Garden. 1286 pp.

BUDOWSKI, G. 1985. Distribución de especies en el bosque tropical húmedo de América a la luz de los procesos de sucesión. Traducido del inglés por Marta Soto. San José (Costa Rica): CATIE. pp. 213-218. 
CARVALHO, J. P. de et al. 1986. Composicao floristica de una mata secundaria no planalto de Belterra no Pará. Anais Simposio do Trópico Umido. Flora y Floresto, V2:197-205. Belem (Brasil): EMBRAPA y CPATU.

FLORES, 5. 1990. Manejo de barbechos antiguos en Brillo Nuevo. En: Agroforesteria Tradicional en la Amazonía Peruana. Jardín Botánico de Nueva York: Denevan y Padoch. Centro de Investigación y Promoción Amazónica. Lima (Perú):

DocumentodeTrabajoNo 11: 12- 146.

FREJTAS, D. 1985. Mapa fisiográfico zona de Jenaro Herrera río Ucayali. Requena (Perú): Centro de Investigaciones de Jenaro Herrera (CIJH).

GENTRY A. 1991. Clave de las principales familias del bosque húmedo tropical. Documento de trabajo. San Louis (Estados Unidos): Missouri Botanical Garden. 104 pp.

KAHN, F. 1983. Architecture comparee deforest tropicales humides et dynamique de la rhizosphere. These Doctuer d' Etat mention Sciences, Academic de Montpellier Universite Des Sciencies et Techniques Du Languedoc. Francia. $426 \mathrm{pp}$.

1990. Clave para diferenciar los géneros de Palmae en la Amazonía a partir del aparato vegetativo. En: Bull. Inst. Fr. études andines 19(2):351-379. Lima (Perú).

OFICINA NACIONAL DE EVALUACION DE RECURSOS NATURALES. 1976. Mapa ecológico del Perú (guía explicativa). Lima. 196 pp.

RIOS, J. 1990. Arboles comunes de los bosques secundarios de Pucallpa (Perú). Proyecto utilización de bosques secundarios en el trópico húmedo peruano. Lima (Perú): UNALM/UT/CIID. 163 pp.

SPICHIGER, R. 1982. Prueba de clave para reconocer, a partir de los órganos vegetativos, las principales familias de árboles de una reserva natural de la Amazonía Peruana. Societé Botanique de Géneve, Suiza. 13: 1-16. Saussurea (Suiza). 
SPICHIGER, R. et al. 1989. Contribución a la flora de la Amazonía Peruana, los árboles del arboretum Jenaro Herrera. Vol. 1. Ginebra (Suiza): Conservatorio y Jardín Botánicos de la ciudad de Ginebra. Organización Suiza para el Desarrollo y la Cooperación e Instituto de Investigaciones de la Amazonía Peruana. $359 \mathrm{pp}$.

1990. Contribución a la flora de la Amazonía Peruana; los árboles del arboretuni Jenaro Herrera. Vol. 2. Ginebra (Suiza): Conservatorio y Jardín Botánicos de la ciudad de Ginebra, Organización Suiza para el Desarrollo y la Cooperación e Instituto de Investigaciones de la Amazonía Peruana. 565 pp.

STOLZE, R. 1981. Fems and fern allies of Guatemala. Part II. Polypodiaceae. En: Fieldiana Botany new series, $\mathrm{N}^{\circ} 6$. Published by Field Museum of Natural History. United States. Publication No 1317. 522 pp.

TRYON, R. M. \& STOLZE, R. 1989. Pteridophyta of Peru Parte II, 13. Pteridaceae

15. Dennstaedtiaceae. Fieldiana, Botany New Series, №22. Publication 1403. Published by Field Museum of Natural History United States. 128 pp.

TRYON, R. et al. 1991. Pteridophyta of Perú. Parte IV. Dryopteridáceae. Fieldiana, Botany New Series, N ${ }^{\circ} 27$, Publication N ${ }^{\circ} 1424$. Published by Field Museum of Natural History United States. 176 pp.

UNIVERSIDAD NACIONAL AGRARIA .LA MOLINA. 1987. Inventario forestal de los bosques secundarios de Pucallpa. San Alejandro. Programa de Investigación: Utilización de bosques secundarios en el trópico húmedo peruano. $103 \mathrm{pp}$.

UNESCO, PNUMA y FAO. 1980. Ecosistemas de los bosques tropicales. Informe sobre el estado de los conocimientos. Paris (Francia). 771 pp.

WEEL, van der. J. J. 1985. Los primeros estados de la regeneración de la vegetación después de la agricultura de corte y quema cerca de Jenaro Herrera en la Amazonía Peruana. Vakgroep Vegetatiekunde, Plantenoecologi e en Onkruidkinde. Wageningen (Holanda). 83 pp. 


\section{Cuadro 2. Relación de especies colectadas y su frecuencia en las vegetaciones secundarias evaluadas.}

\begin{tabular}{|c|c|c|c|c|c|c|c|c|c|c|}
\hline \multirow{2}{*}{$\begin{array}{l}\text { Familia } \\
\text { Nombre Científico }\end{array}$} & \multirow{2}{*}{$\begin{array}{l}\text { Nombre } \\
\text { Vernacular }\end{array}$} & \multirow{2}{*}{$\begin{array}{l}\text { Número } \\
\text { de } \\
\text { Colecta }\end{array}$} & \multicolumn{8}{|c|}{ Edad de la Purma } \\
\hline & & & 1 & 3 & 5 & 8 & 10 & 12 & 14 & 17 \\
\hline $\begin{array}{l}\text { ACANTHACEAE } \\
\text { Mendoncia smithii Leonard } \\
\text { Mendoncia sp. }\end{array}$ & $\begin{array}{l}\text { Soga } \\
\text { Soga }\end{array}$ & $\begin{array}{l}078 \\
221\end{array}$ & & $\mathrm{x}$ & & & $\mathrm{x}$ & & & \\
\hline $\begin{array}{l}\text { ANARCARDIACEAE } \\
\text { Mangifera indica L. } \\
\text { Tapirira guianensis Aublet }\end{array}$ & $\begin{array}{l}\text { Mangua } \\
\text { Wira casppi }\end{array}$ & $\begin{array}{l}168 \\
274,370\end{array}$ & & & & $\mathrm{x}$ & & $\mathrm{x}$ & & \\
\hline $\begin{array}{l}\text { ANNONACEAE } \\
\text { Bagageopsis mattogrossensis } \\
\text { (R.E. Fries) R.E Fries } \\
\text { Dicljncjnona tess,nannii Diels } \\
\text { Duguetia spixiana Mart. } \\
\text { Guatteria citriodova Ducke } \\
\text { Guatteria hyposericea Diels } \\
\text { Guatteria megalophylla Diels } \\
\\
\text { Oxandra sp. } \\
\text { Unonopsís sp. } \\
\text { Xylopia bentamii RE. Fries } \\
\text { Xylopia sp. }\end{array}$ & $\begin{array}{l}\text { Icoja } \\
\text { tortuga caspi } \\
\text { tortuga caspi } \\
\text { carahuasca } \\
\text { carahuasca } \\
\text { espintana hoja } \\
\text { grande. } \\
\text { machin sapote } \\
\text { tortuga caspi } \\
\text { espintana pinsha } \\
\text { gallo }\end{array}$ & $\begin{array}{l}365 \\
285 \\
366 \\
099,278,3 \\
30 \\
155,349 \\
182,376 \\
216 \\
354 \\
359 \\
275\end{array}$ & & $\mathrm{x}$ & & $\mathrm{x}$ & $\begin{array}{l}\mathrm{X} \\
\mathrm{x}\end{array}$ & $\mathrm{X}$ & $\mathrm{x}$ & $\begin{array}{l}\mathrm{x} \\
\mathrm{x} \\
\mathrm{x} \\
\mathrm{x} \\
\mathrm{x} \\
\mathrm{x}\end{array}$ \\
\hline $\begin{array}{l}\text { APOCYNACEAE } \\
\text { Aspidosperma sp. } \\
\text { Macoubea sprucei } \\
\text { (MuelI. Arg.) } \\
\text { Markgraf } \\
\text { Parac/iancornia peruviana } \\
\text { Monachino }\end{array}$ & $\begin{array}{l}\text { quillobordón } \\
\text { yahuar huayo } \\
\text { colorado } \\
\text { naranjo podrido. }\end{array}$ & $\begin{array}{l}197 \\
202 \\
190\end{array}$ & & & & & $\begin{array}{l}\mathrm{x} \\
\mathrm{x} \\
\mathrm{x}\end{array}$ & & & \\
\hline $\begin{array}{l}\text { ARACEAE } \\
\text { Anthurium sp. } \\
\text { Monstera obliqua Miq. } \\
\text { Monstera sp. } \\
\text { Philodendrom deflexum } \\
\text { Poepp. } \\
\text { ex Schott } \\
\text { Philodendron aff. deltoideum } \\
\text { Poepp. }\end{array}$ & $\begin{array}{l}\text { itininga } \\
\text { itininga } \\
\text { itininga } \\
\text { itininga hoja } \\
\text { grande } \\
\text { itininga }\end{array}$ & $\begin{array}{l}118 \\
083 \\
315 \\
\\
150\end{array}$ & & $\mathrm{x}$ & $\mathrm{x}$ & & & $\mathrm{x}$ & & \\
\hline
\end{tabular}




\section{Continua...}

\begin{tabular}{|c|c|c|c|c|c|c|c|c|c|c|}
\hline \multirow{2}{*}{$\begin{array}{l}\text { Familia } \\
\text { Nombre Científico }\end{array}$} & \multirow{2}{*}{$\begin{array}{l}\text { Nombre } \\
\text { Vernacular }\end{array}$} & \multirow{2}{*}{$\begin{array}{l}\text { Número de } \\
\text { Colecta }\end{array}$} & \multicolumn{8}{|c|}{ Edad de la Purma } \\
\hline & & & 1 & 3 & 5 & 8 & 10 & 12 & 14 & 17 \\
\hline $\begin{array}{l}\text { ARACEAE } \\
\text { Astrocryurn chambira Buffet } \\
\text { Attalea spectabilis Martius } \\
\text { Bactris sp } \\
\text { Chamaedorea sp. } \\
\text { Desmoncus vacivus L. Bailey } \\
\text { Geonoma acaulis C. Martius } \\
\text { Oenoca pus bataua C. Martius } \\
\text { Socratea exorrhiza C. Martius) } \\
\text { H. A. Wendland } \\
\text { Wettinia augusta } \\
\text { Poeppig \& Endl. }\end{array}$ & $\begin{array}{l}\text { chambira } \\
\text { catirina } \\
\text { ñejilla } \\
\text { palmichillo } \\
\text { casha vara } \\
\text { palmichillo } \\
\text { ungurahui } \\
\text { casha pona } \\
\text { ponilla }\end{array}$ & $\begin{array}{l}400 \\
232,268, \\
314 \\
385,077, \\
278, \\
319,327 \\
125 \\
263 \\
280 \\
356,184, \\
240,356 \\
353 \\
161\end{array}$ & $\mathrm{x}$ & $\mathrm{x}$ & $\mathrm{x}$ & $\mathrm{x}$ & $\mathrm{x}$ & $\begin{array}{l}\mathrm{x} \\
\mathrm{x} \\
\mathrm{x} \\
\mathrm{x} \\
\mathrm{x}\end{array}$ & $\mathrm{x}$ & $\begin{array}{l}\mathrm{x} \\
\mathrm{x}\end{array}$ \\
\hline $\begin{array}{l}\text { ASCLEPIADACEAE } \\
\text { Blepharodom sp }\end{array}$ & soga & 222 & & & & $\mathrm{x}$ & & & & \\
\hline $\begin{array}{l}\text { ASTERACEAE } \\
\text { Mikania banisteridae DC. } \\
\text { Mikania sp. }\end{array}$ & $\begin{array}{l}\text { soga } \\
\text { soga }\end{array}$ & $\begin{array}{l}064,186,30 \\
4 \\
336\end{array}$ & & $\mathrm{x}$ & & & $\mathrm{x}$ & & $\mathrm{x}$ & $\mathrm{x}$ \\
\hline $\begin{array}{l}\text { BIGNONIACEAE } \\
\text { Arrabiacea cinnamomea } \\
\text { (A.DC) Sw. } \\
\text { Arrabidaea aff. Florida } \\
\text { A. DC. } \\
\text { Jacaranda copaia subsp. } \\
\text { spectabilis (C. Martius ex } \\
\text { A. DC.) A. Gentry } \\
\text { Tynanthus panurensis } \\
\text { (Bur) Sw }\end{array}$ & $\begin{array}{l}\text { soga } \\
\text { clavo huasca } \\
\text { huamansamana } \\
\text { clavo huasca }\end{array}$ & $\begin{array}{l}259 \\
332 \\
062,261 \\
091\end{array}$ & & $\mathrm{x}$ & $\mathrm{x}$ & & & $\mathrm{x}$ & & $\mathrm{x}$ \\
\hline $\begin{array}{l}\text { BORAGINECEAE } \\
\text { Cordia nodosa lamarck }\end{array}$ & Hormiga caspi & 159,198 & & & & & $\mathrm{x}$ & $\mathrm{x}$ & & \\
\hline
\end{tabular}




\section{...continua}

\begin{tabular}{|c|c|c|c|c|c|c|c|c|c|c|}
\hline \multirow{2}{*}{$\begin{array}{l}\text { Familia } \\
\text { Nombre Científico }\end{array}$} & \multirow{2}{*}{$\begin{array}{l}\text { Nombre } \\
\text { Vernacular }\end{array}$} & \multirow{2}{*}{$\begin{array}{l}\text { Número de } \\
\text { Colecta }\end{array}$} & \multicolumn{8}{|c|}{ Edad de la Purma } \\
\hline & & & 1 & 3 & 5 & 8 & 10 & 12 & 14 & 17 \\
\hline $\begin{array}{l}\text { BURSERACEAE } \\
\text { Protium apiculatum Swart } \\
\text { Trattinnickia aspera (Standley) } \\
\text { Swart } \\
\text { Trattinnickia } \text { sp. }\end{array}$ & $\begin{array}{l}\text { lacre } \\
\text { gallinazo } \\
\text { copal } \\
\text { copal }\end{array}$ & $\begin{array}{l}133 \\
090,331 \\
101\end{array}$ & & & $\begin{array}{l}x \\
x\end{array}$ & & & & $\mathrm{x}$ & \\
\hline $\begin{array}{l}\text { CARYOCARIACEAE } \\
\text { Caryocar glabrum (Aubl.) Pers } \\
\text { Subs. glabrum }\end{array}$ & almendro & 218,321 & & & & & $\mathrm{x}$ & & $\mathrm{x}$ & \\
\hline $\begin{array}{l}\text { CEPROPIACEAE } \\
\text { Cecropia ficifolia Warburg ex } \\
\text { Snethage } \\
\text { Cecropia sciadophylla } \\
\text { C.Mart. } \\
\text { Pourouma cecropiafolia } \\
\text { C.Martius } \\
\text { Pourourna mollis Trécul } \\
\text { Pourourna sp. }\end{array}$ & $\begin{array}{l}\text { cetico blanco } \\
\text { cetico color } \\
\text { uvilla } \\
\text { sacha uvilla } \\
\text { sacha uvilla }\end{array}$ & $\begin{array}{l}388,111,151 \\
308,343 \\
074,112,160 \\
121 \\
322,357 \\
178,272\end{array}$ & $\mathrm{x}$ & $\mathrm{x}$ & $x$ & $\mathrm{x}$ & $\mathrm{x}$ & $\mathrm{x}$ & $\mathrm{x}$ & $x$ \\
\hline $\begin{array}{l}\text { CELASTRACEAE } \\
\text { Goupia sp. }\end{array}$ & Casearia & 069,211 & & & $\mathrm{x}$ & & $\mathrm{x}$ & & & \\
\hline $\begin{array}{l}\text { CLUSIACEAE } \\
\text { Clussía loretensis Engler } \\
\text { Garcinia madruno } \\
\text { (Kunth) Hammel } \\
\text { Rheedia gardneriana P1.\& Tr. } \\
\text { Tovomita sp. } \\
\text { Vismia angusta Miquel } \\
\text { Vismia gracilis Hieronynus } \\
\text { Vismia ferrugenea H. } 8 \text {.K. } \\
\text { Vismia tomentosa R. \& P. }\end{array}$ & $\begin{array}{l}\text { chulla chaqui } \\
\text { charichuelo } \\
\text { charíchuelo } \\
\text { chulla chaqui } \\
\text { pichirina } \\
\text { h. ancha. } \\
\text { pichirina } \\
\text { h.menuda } \\
\text { pichirina } \\
\text { h.menuda } \\
\text { pichirina } \\
\text { h.menuda }\end{array}$ & $\begin{array}{l}058 \\
213 \\
375 \\
230 \\
054,124,170 \\
055,114,171, \\
320 \\
210,350 \\
097,309,379\end{array}$ & & $\mathrm{x}$ & $\mathrm{x}$ & $\mathrm{x}$ & $\mathrm{x}$ & & $\mathrm{x}$ & $\begin{array}{l}\mathrm{x} \\
\mathrm{x}\end{array}$ \\
\hline
\end{tabular}




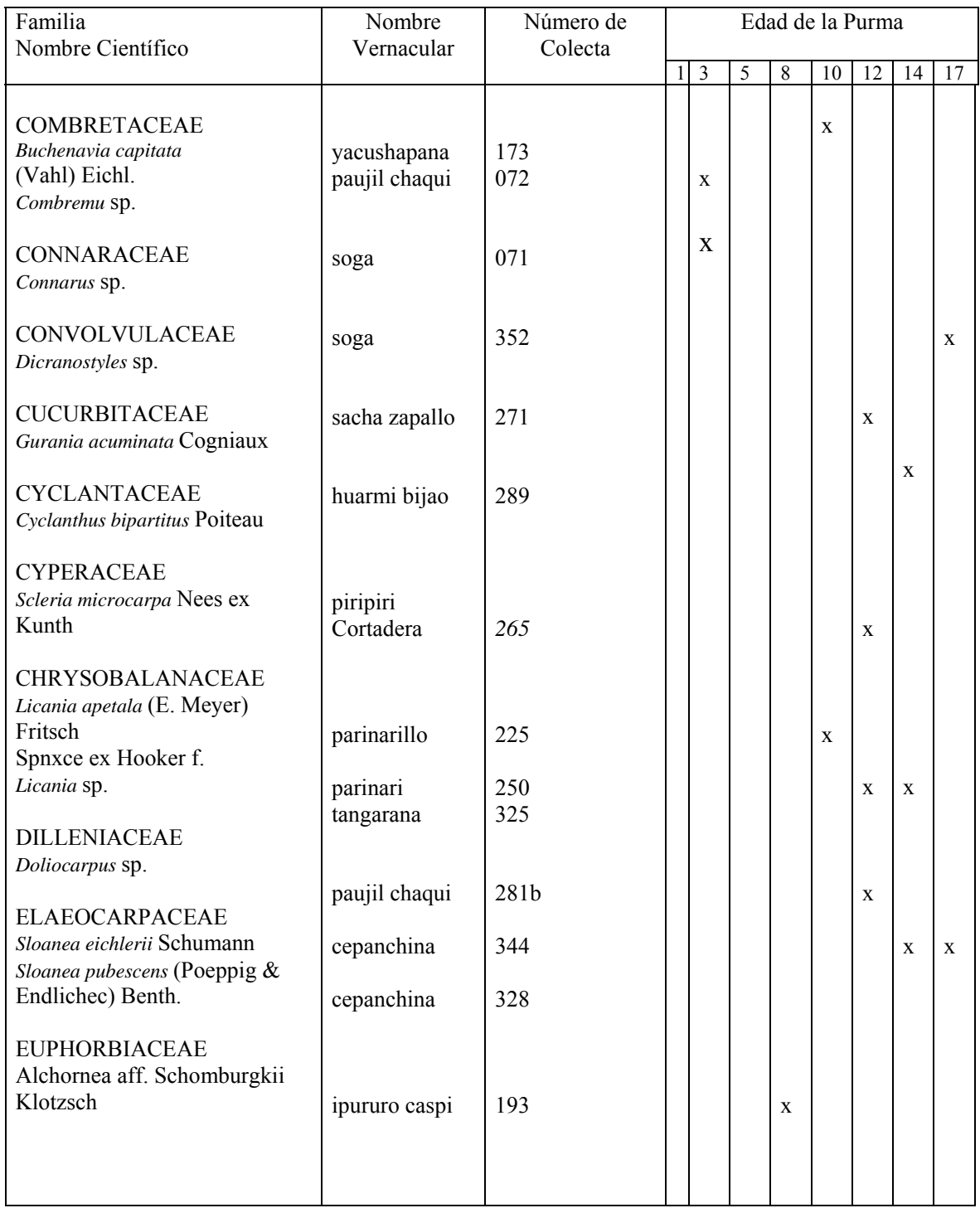




\section{.Continua}

\begin{tabular}{|c|c|c|c|c|c|c|c|c|c|c|}
\hline Familia & Nombre & Número de & & & Eda & de & la $\mathrm{P}$ & $\mathrm{rma}$ & & \\
\hline & & & 1 & 3 & 5 & 8 & 10 & 12 & 14 & 17 \\
\hline $\begin{array}{l}\text { Alchornea triplinervia } \\
\text { (Sprengel) Muell Arg. } \\
\text { Alchorneopsis floribunda } \\
\text { (Bentb.) Muell. Arg } \\
\text { Áparisthmium cordaturn } \\
\text { (Adr. Juss.) Baillon } \\
\text { Dalec/iarnpia dioscoreifo1ia } \\
\text { Poeppig } \\
\text { Mabea maynensis Spruce } \\
\text { FABACEAE } \\
\text { Dalbergia monetaria L.f. } \\
\text { Enterolobiom sp. } \\
\text { Inga alba (Swartz) } \\
\text { Willdenow } \\
\text { Inga laurina (Swartz) } \\
\text { Willdenow } \\
\text { Inga lopadadenia Harms } \\
\text { Inga punctata Willdenow } \\
\text { Inga ricardorum Bernardi } \\
\text { \& Spichiger } \\
\text { Inga thibaudiana DC. } \\
\text { Inga sp. } \\
\text { Inga sp. } \\
\text { Lonchocarpus sp. } \\
\text { Machaerium Iatifolium Rusby } \\
\text { Macherium sp. } \\
\text { Macherium sp. } \\
\text { Macherium sp. } \\
\text { Mimosa rufescens Bentham } \\
\text { Parkia multijuga Bentham } \\
\text { Tachigali melinonii Harms } \\
\text { Swartzia cardiosperma Spruee } \\
\text { ex Bentham }\end{array}$ & $\begin{array}{l}\text { zancudo caspi } \\
\text { zancudo caspi } \\
\text { yanabara } \\
\text { soga } \\
\text { shiringuilla } \\
\\
\text { sacha barbasco } \\
\text { pashaco } \\
\text { shimbillo } \\
\text { shimbillo } \\
\text { shimbillo } \\
\text { shimbillo } \\
\text { shimb. alado } \\
\text { shimbillo } \\
\text { shimbillo } \\
\text { shimbillo } \\
\text { colorado } \\
\text { barbasco } \\
\text { del monte soga } \\
\text { soga } \\
\text { soga } \\
\text { soga } \\
\text { uña de gato } \\
\text { pashaquillo } \\
\text { pashaco curtido } \\
\text { tangarana } \\
\text { marimari }\end{array}$ & $\begin{array}{l}068,098,212, \\
288,311,340 \\
105,113 \\
100 \\
185,351 \\
347 \\
\\
405,346 \\
126 \\
179 \\
404 \\
081 \\
176,273,297 \\
395 \\
369 \\
241,329 \\
082 \\
085 \\
066 \\
067 \\
237 \\
391,096 \\
258 \\
362 \\
283 \\
238 \\
\\
\\
\end{array}$ & $\mathrm{x}$ & $\begin{array}{l}X \\
X\end{array}$ & $\mathrm{x}$ & & $\mathrm{x}$ & $\mathrm{x}$ & $\mathrm{x}$ & $\begin{array}{l}x \\
x\end{array}$ \\
\hline
\end{tabular}




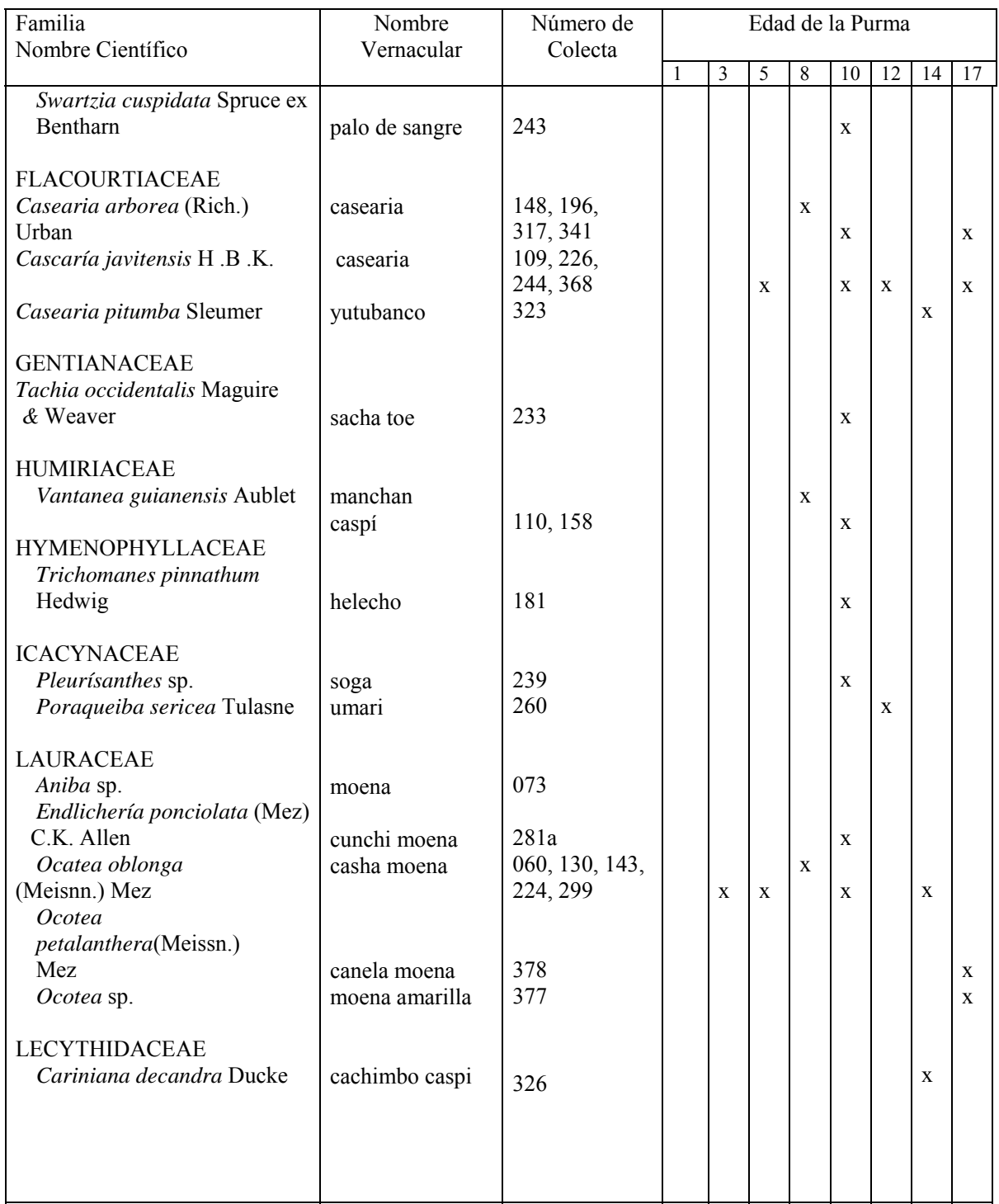




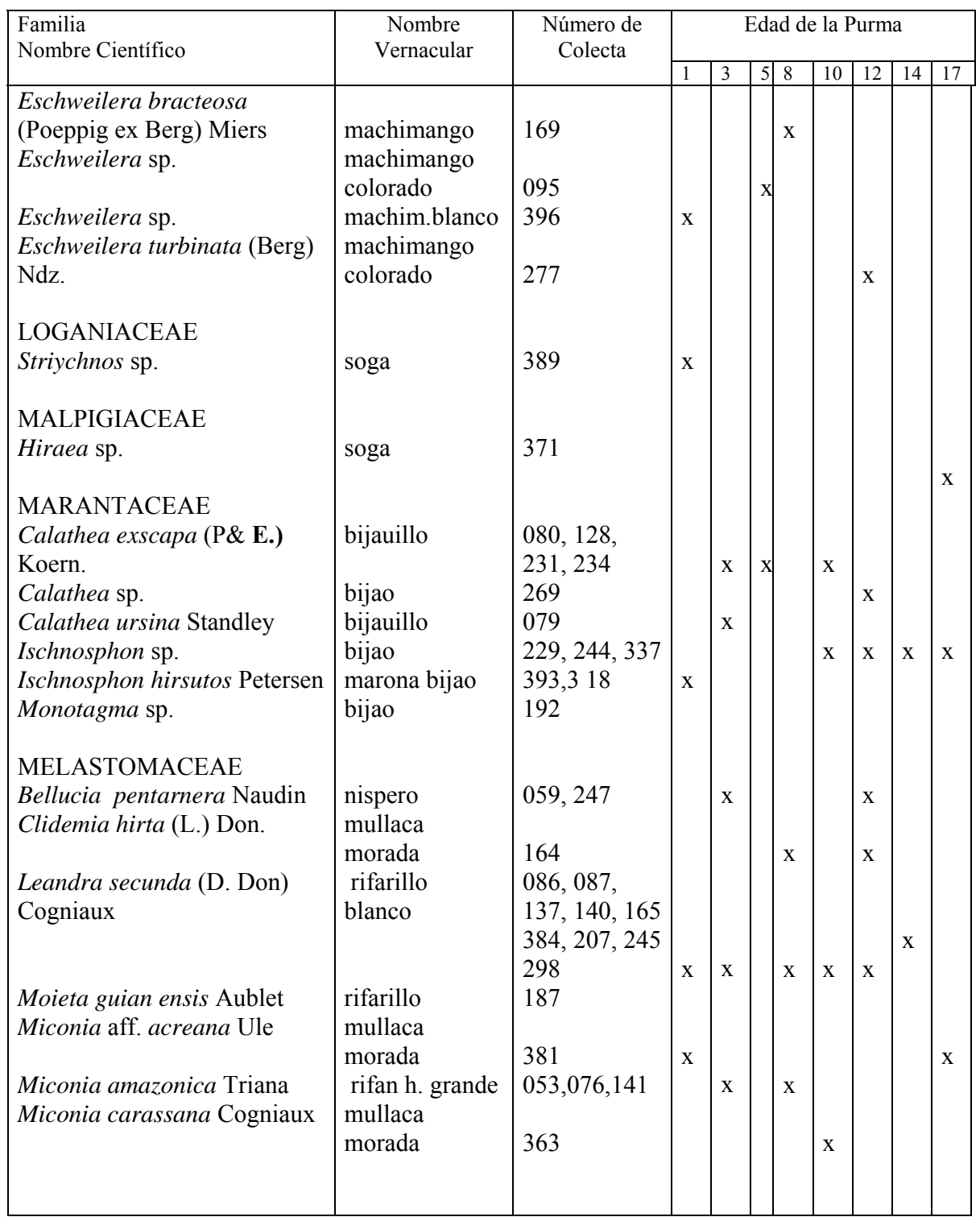




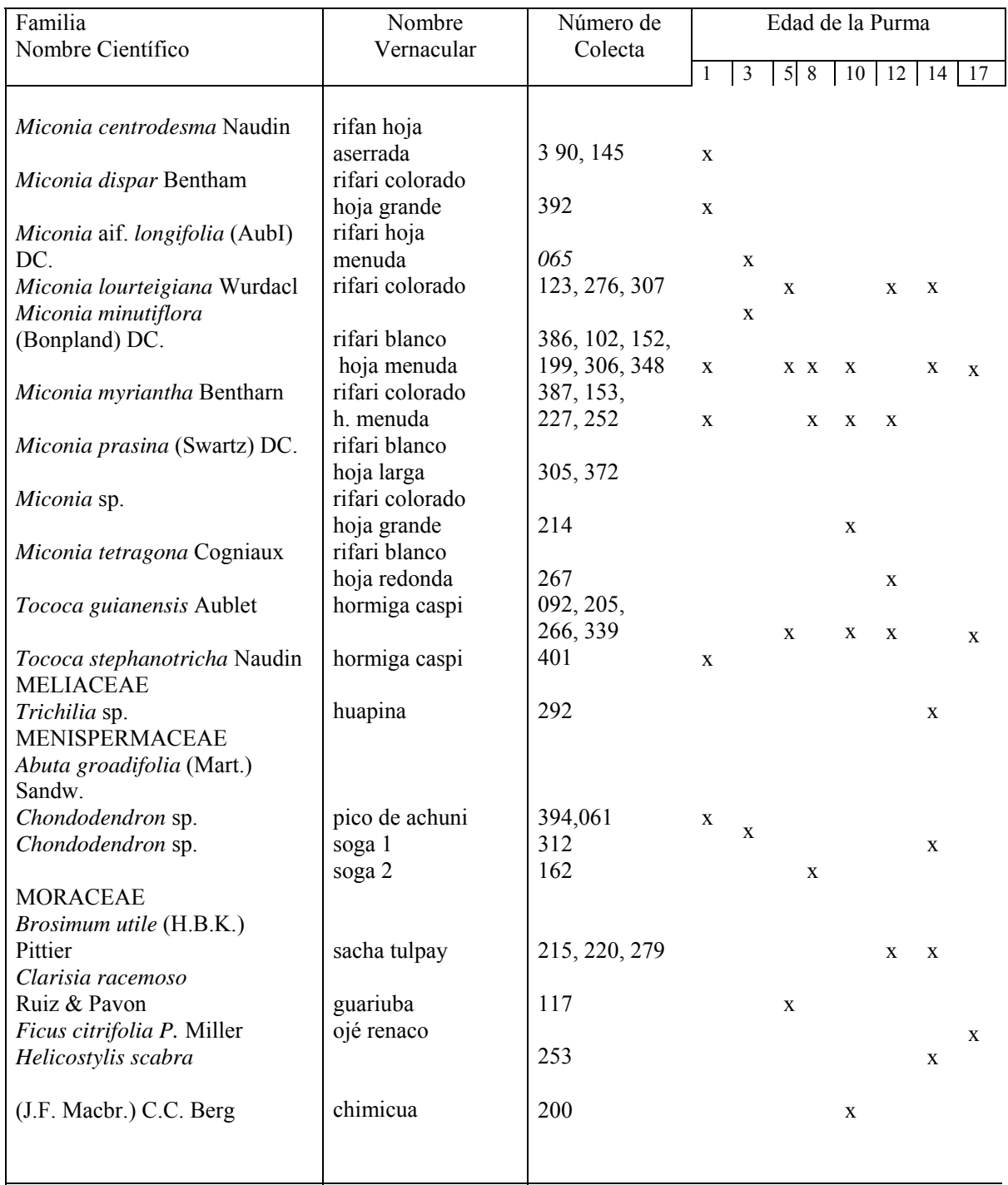




\begin{tabular}{|c|c|c|c|c|c|c|c|c|c|c|}
\hline \multirow{2}{*}{$\begin{array}{l}\text { Familia } \\
\text { Nombre cientifico }\end{array}$} & \multirow{2}{*}{$\begin{array}{l}\text { Nombre } \\
\text { Vernacular }\end{array}$} & \multirow{2}{*}{$\begin{array}{l}\text { Número de } \\
\text { Colecta }\end{array}$} & \multicolumn{8}{|c|}{ EDAD DE LA PURMA (Años) } \\
\hline & & & 1 & 3 & 5 & 8 & 10 & 12 & 14 & 17 \\
\hline $\begin{array}{l}\text { Olmedia aspera R. \& P. } \\
\text { Pseudolmedia laevigata Trécul } \\
\text { Pseudolmedia rnacrophylla } \\
\text { Trécul } \\
\text { Tiymatococcus aniazonicus } \\
\text { Poeppig \& Endlicher } \\
\text { MUSACEAE } \\
\text { Heliconia hirsuta L.f. }\end{array}$ & $\begin{array}{l}\text { chimicua sacba } \\
\text { tulpay } \\
\text { chimicua } \\
\text { chimieua } \\
\text { situlh }\end{array}$ & $\begin{array}{l}188,335 \\
177,180 \\
122 \\
147 \\
138,255,355\end{array}$ & & & $\mathrm{x}$ & $\mathrm{x}$ & $\begin{array}{l}\mathrm{x} \\
\mathrm{x}\end{array}$ & $\mathrm{x}$ & & $\mathrm{x}$ \\
\hline $\begin{array}{l}\text { MYRISTICACEAE } \\
\text { Osteophloceum platyspermum } \\
\text { (ADC.) Warburg } \\
\text { Virola mollisima (A.DC.) } \\
\text { Warb. } \\
\text { Virola sp. }\end{array}$ & $\begin{array}{l}\text { cumala blanca } \\
\text { cumala } \\
\text { cumala blanca }\end{array}$ & $\begin{array}{l}094 \\
\\
163 \\
156\end{array}$ & & & $\mathrm{x}$ & $\begin{array}{l}x \\
x\end{array}$ & & & & $\mathrm{x}$ \\
\hline $\begin{array}{l}\text { MYRSINACEAE } \\
\text { Cybianthus sp. }\end{array}$ & balata rosada & 294 & & & & & & & $\mathrm{x}$ & \\
\hline $\begin{array}{l}\text { MYRTACEAE } \\
\text { Eugenia sp. } \\
\text { Eugenia aff. tetrasticha } \\
\text { Poeppig ex O. Berg } \\
\text { Myrcia fallax (Richard) DC. } \\
\text { Myrcia sp. }\end{array}$ & $\begin{array}{l}\text { sacha guayaba } \\
\text { sacha guayaba } \\
\text { lanza caspi } \\
\text { sanango }\end{array}$ & $\begin{array}{l}358 \\
\\
364 \\
313 \\
136\end{array}$ & & & & & $x$ & & $\mathrm{x}$ & $\mathrm{x}$ \\
\hline $\begin{array}{l}\text { NYCTAGINACEAE } \\
\text { Neea sp. }\end{array}$ & muesca huayo & 093,284 & & & $\mathrm{x}$ & & & $\mathrm{x}$ & & \\
\hline $\begin{array}{l}\text { OLACACEAE } \\
\text { Dulacia candida (Poeppig) } \\
\text { Kuntze } \\
\text { ONAGRACEAE } \\
\text { Ludwigia nervosa (Poiret) H. } \\
\text { Hara }\end{array}$ & sacha guayaba & 183 & & & & & & $x$ & & \\
\hline
\end{tabular}




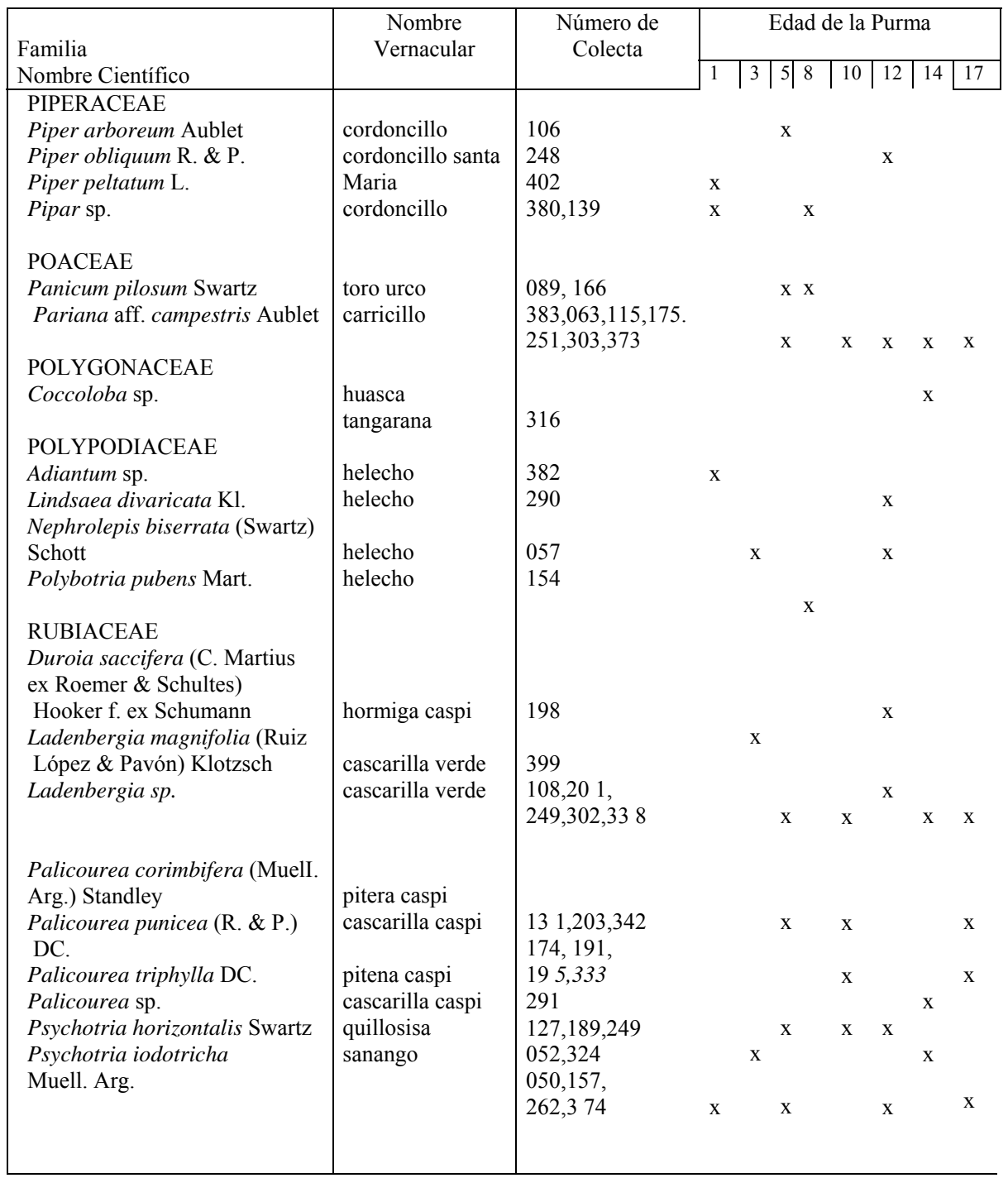




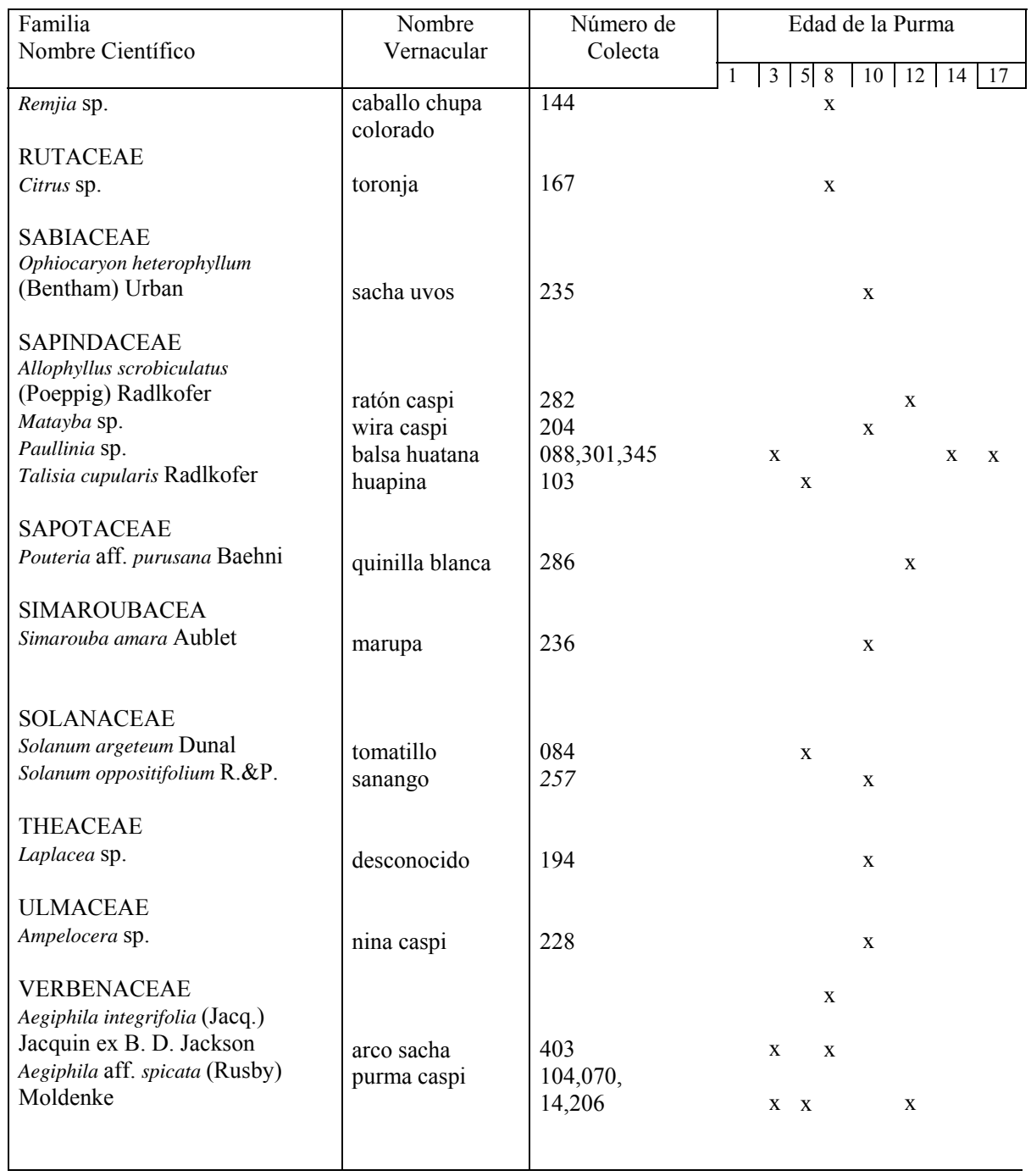




\section{...Contiúa}

\begin{tabular}{|c|c|c|c|c|c|c|c|c|c|c|}
\hline \multirow{2}{*}{$\begin{array}{l}\text { Familia } \\
\text { Nombre Científico }\end{array}$} & \multirow{2}{*}{$\begin{array}{l}\text { Nombre } \\
\text { Vernacular }\end{array}$} & \multirow{2}{*}{$\begin{array}{l}\text { Numero de } \\
\text { Colecta }\end{array}$} & \multicolumn{8}{|c|}{ Edad de Purma } \\
\hline & & & 1 & 3 & 5 & 8 & 10 & 12 & 14 & 17 \\
\hline $\begin{array}{l}\text { VIOLACEAE } \\
\text { Leonia cymosa C. Martius } \\
\text { Rinorea racemosa (C. Martius) }\end{array}$ & $\begin{array}{l}\text { Leonia } \\
\text { trompetero } \\
\text { caspi }\end{array}$ & $\begin{array}{l}149,209 \\
256\end{array}$ & & & & $\mathrm{X}$ & $X$ & $\mathrm{X}$ & & \\
\hline $\begin{array}{l}\text { OCHYSIACEAE } \\
\text { Erismleonia trompetero caspi }\end{array}$ & quillo sisa & 242 & & & & & $X$ & & & \\
\hline
\end{tabular}

Figura 1. Parcelas de muestreo (a) parcela mayor, (b) sub parcelas Donde:

A: corresponde a un cuadrado de 2,5 x 2,5 para inventario de plantas de $0,1 \mathrm{ma} 1,30 \mathrm{~m}$ de altura.

B: corresponde a un cuadrado de 2,5 x 2,5 para inventario de plantas mayores a $1,31 \mathrm{~m}$ de altura y Dap menores de $10 \mathrm{~cm}$.

$\mathrm{C}$ : corresponde a un cuadrado de $10 \times 10 \mathrm{~m}$, para inventario de plantas mayores a $1,31 \mathrm{~m}$ de altura y Dap mayores de $10 \mathrm{~cm}$.
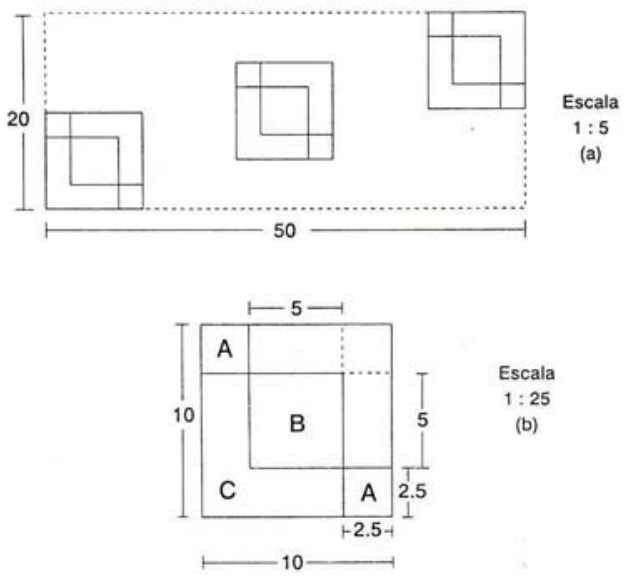
Figura 2. Diagrama de la descomposición Florística por Taxones

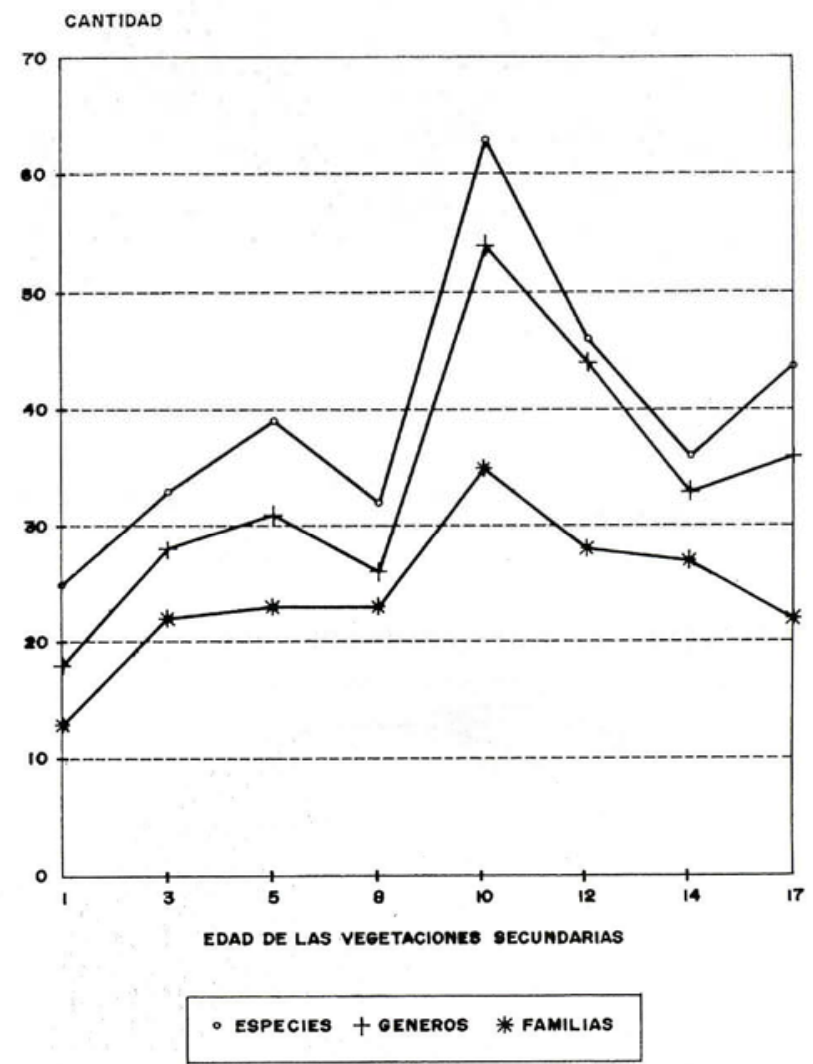


Figura 3. Familias por mayor abundancia de especies

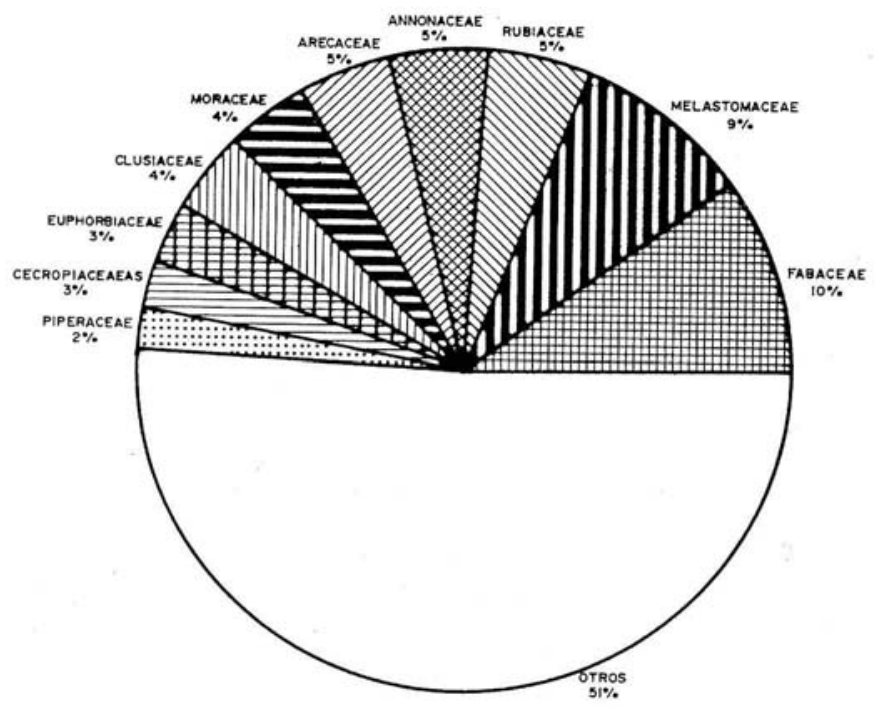

Figura 4. Familias por mayor número de individuos

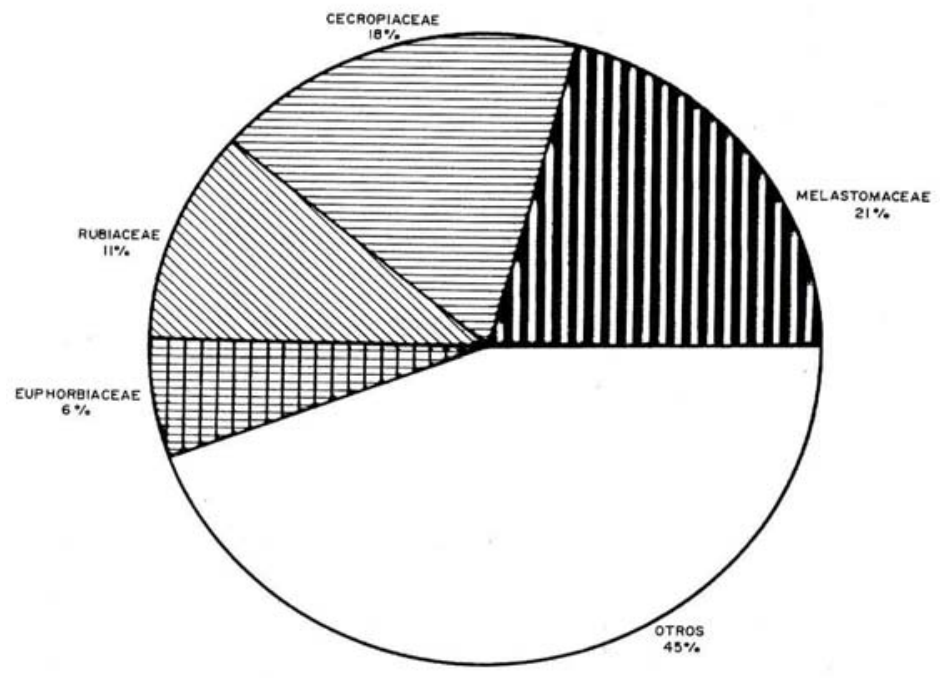

